\title{
Galectin-3 and fibulin-1 in systolic heart failure - relation to glucose metabolism and left ventricular contractile reserve
}

Pernille Holmager ${ }^{1,2,11^{*}}$, Michael Egstrup ${ }^{3}$, Ida Gustafsson ${ }^{4}$, Morten Schou 2,5 , Jordi S. Dahl ${ }^{6}$, Lars Melholt Rasmussen ${ }^{7,8}$, Jacob E. Møller ${ }^{9}$, Christian Tuxen ${ }^{10}$, Jens Faber ${ }^{1,2}$ and Caroline Kistorp ${ }^{1,2}$

\begin{abstract}
Background: Heart failure (HF) patients with diabetes (DM) have an adverse prognosis and reduced functional capacity, which could be associated with cardiac fibrosis, increased chamber stiffness and reduced left ventricular (LV) contractile reserve. Galectin-3 (Gal-3) and fibulin-1 are circulating biomarkers potentially reflecting cardiac fibrosis. We hypothesize that plasma levels of Gal-3 and fibulin-1 are elevated in HF patients with DM and are associated with reduced LV contractile reserve in these patients.

Methods: A total of 155 patients with HF with reduced ejection fraction underwent a low-dose dobutamine echocardiography and blood sampling for biomarker measurements. Patients were classified according to history of DM and an oral glucose tolerance test (OGTT) as: normal glucose tolerance (NGT) $(n=70)$, impaired glucose tolerance $(\mathrm{IGT})(n=25)$ and DM $(n=60)$.

Results: Galectin-3 levels were elevated in DM patients as compared to non-diabetic patients $(P=0.02)$, while higher fibulin-1 levels were observed in HF patients with IGF and DM $(P=0.07)$. Reduced LV contractile reserve was associated with increasing Gal-3 levels $(\beta=-0.19, P=0.03$ ) although, this association was attenuated after adjustment for estimated glomerular filtration rate $(P=0.66)$. Fibulin-1 was not associated with LV contractile reserve $(P=0.71)$.

Conclusions: Galectin-3 and fibulin-1 levels were elevated in HF patients with impaired glucose metabolism. However, reduced LV contractile reserve among HF patients with DM does not to have an independent impact on plasma Gal-3 and fibulin-1 levels.
\end{abstract}

Keywords: Diabetes, Myocardial fibrosis, Galectin-3, Fibulin-1, Left ventricular contractile reserve

\section{Background}

Patients with heart failure with reduced ejection fraction (HFrEF) and concomitant diabetes (DM) have a considerably increased mortality rate and reduced exercise capacity compared to non-diabetic HFrEF patients [1]. It is well-established that in HFrEF DM is frequent, and screening with an oral glucose tolerance test (OGTT) reveals a prevalence of more than $30 \%$ [2]. We have previously reported that the co-existence of DM, whether it is by history or newly diagnosed is associated with a

\footnotetext{
* Correspondence: holmager@dadlnet.dk

'Department of Medicine, Endocrine Unit, Herlev University Hospital, Herlev, Denmark

${ }^{2}$ Faculty of Health and Medical Sciences, Copenhagen University, Copenhagen, Denmark

Full list of author information is available at the end of the article
}

reduced left ventricular (LV) contractile reserve assessed by low-dose dobutamine echocardiography (LDDE) among HFrEF patients [3]. Further, reduced LV contractile reserve is predictive of prognosis and thus could contribute to the unfavorable outcome of diabetic HFrEF patients [4]. The pathophysiological processes leading to reduced LV contractile reserve remain to be fully elucidated but have been associated with myocardial fibrosis in patients with cardiomyopathy [5].

Galectin-3 (Gal-3) is a galactoside-binding protein which is released to the circulation by activated macrophages and is considered a biomarker of cardiac fibrosis and remodeling [6]. In the myocardium, Gal-3 is primarily expressed in the fibroblasts and in macrophages and plays an important role in the formation of myocardial fibrosis 
through activation of fibroblasts [6]. In DM increased plasma Gal-3 levels have been associated with both macro- and microvascular complications [7]. Moreover, high levels of Gal-3 are reported in patients with chronic HFrEF and renal dysfunction [8]. Hence, we hypothesize that elevated Gal-3 is associated with reduced LV contractile reserve found among HFrEF patients with DM.

The novel promising biomarker fibulin-1 is emerging as a potential biomarker of cardiomyopathy [9]. Fibulin1 is an extracellular matrix glycoprotein and is considered to reflect pathological accumulation of extracellular matrix components surrounding vascular smooth muscle cells [10]. To our knowledge no previous studies of fibulin-1 in HFrEF patients have been performed. In patients with chronic renal dysfunction high levels of fibulin-1 are associated with increasing age, HbA1c and creatinine [11]. Plasma fibulin-1 levels have been investigated in patients with aortic stenosis, where an association with decreased longitudinal systolic LV function has been observed [12]. The effects of HFrEF and abnormal glucose metabolism on circulating fibulin-1 levels have, to our knowledge, not been examined previously.

Therefore, the aims of this study were to evaluate the impact of DM and abnormal glucose metabolism on plasma Gal-3 and fibulin-1 concentrations, and further to investigate whether Gal-3 and fibulin-1 levels were associated with LV contractile reserve in HFrEF patients according to presence of DM.

\section{Methods}

\section{Study population}

Between May 2008 and June 2010155 patients were recruited from the HF clinic at Frederiksberg University Hospital, Copenhagen, Denmark. The cohort has previously been described in detail [3]. Patients were enrolled in the present study if LV ejection fraction (LVEF) was $\leq 45 \%$ by echocardiography and baseline measurements of Gal-3 and fibulin-1 were available.

Patients were classified as having DM either by history or DM newly diagnosed by an oral glucose tolerance test (OGTT). The OGTT was performed in patients without known DM within 2 weeks after the baseline visit by ingestion of $75 \mathrm{~g}$ glucose dissolved in $250 \mathrm{~mL}$ water. Plasma glucose was measured in the fasting state (fasting plasma glucose, FPG) and after 2 h (2hPG). Glycemic status was defined according to WHO diagnostic criteria: Normal glucose tolerance (NGT): FPG $<6.1 \mathrm{mmol} / \mathrm{L}$ and $2 \mathrm{hPG}$ $<7.8 \mathrm{mmol} / \mathrm{L}$. Impaired glucose tolerance (IGT): FPG $<7.0 \mathrm{mmol} / \mathrm{L}$ and $2 \mathrm{hPG}$ between 7.8 and $11.1 \mathrm{mmol} / \mathrm{L}$. DM: FPG $>7.0 \mathrm{mmol} / \mathrm{L}$ and/or $2 \mathrm{hPG}>11.1 \mathrm{mmol} / \mathrm{L}$.

\section{Echocardiography}

Resting echocardiography and LDDE (as described in detail previously [3]) was performed within 2 weeks after referral to the outpatient clinic on a standard ultrasound machine (IE-33, Philips Inc.) and stored for later analysis [3]. The Doppler analyses were based on an average of five beats for patients with sinus rhythm and 10 beats for patients with atrial fibrillation (AF). LVEF was calculated by the biplane method from apical four- and twochamber views [13].

\section{Laboratory analysis}

After a minimum 8-h overnight fast and 20 min of supine rest, venous blood was obtained for measurement. Blood was drawn into EDTA tubes, promptly centrifuged at $4{ }^{\circ} \mathrm{C}$, and plasma was frozen at $-80{ }^{\circ} \mathrm{C}$ in aliquots until laboratory analyses were performed. Insulin resistance was estimated using the Homeostasis Model of Assessment-Insulin Resistance (HOMA-IR): Fasting Glucose $(\mathrm{mmol} / \mathrm{L})$ x Insulin $(\mathrm{mU} / \mathrm{L}) / 22.5$. Galectin-3 was determined using an ELISA kit (BG Medicine, Waltham, USA) [14]. The lower detection limit was $1.32 \mathrm{ng} / \mathrm{mL}$, intra- and interassay coefficient of variation (CV) $<6 \%$ and $<10 \%$, respectively [14]. The fibulin-1 double-antibody sandwich ELISA has previously been described extensively [15]. Antibodies are a mouse antifibulin monoclonal 5D12/H7 IgG and goat anti-rabbit IgG alkaline phosphatase [16]. The assay has a lower detection limit of $1.56 \mu \mathrm{g} / \mathrm{mL}$, an intra- and interassay $\mathrm{CV}<8 \%$ and $\sim 10 \%$, respectively [15].

\section{Statistical analysis}

Distribution of the biomarkers Gal-3 and fibulin-1 in the total study population is depicted in histograms. Continuous variables are presented as mean (SD) or as median (interquartile range), as appropriate. The groups were compared using independent sample $T$-test or Kruskall-Wallis' test if the assumption of Gaussian distribution was not met. Fibulin-1 levels were normally distributed, while GAL-3 levels were not. The IGT group was small, and we previously demonstrated that LV contractile reserve is reduced in patients with known or new DM but not affected by IGT. Hence, we divided the population in two groups: DM (new or known), and non-DM (NGT or IGT) in the multivariable linear regression analysis. The associations between Gal-3, fibulin- 1 and $\mathrm{HbA1c}$ were depicted in scatter plots. Multivariable linear regression analyses examining the association between Gal-3 and fibulin-1 levels with LV function were performed. The multivariable regression analyses included co-variables considered of having potential impact of $\mathrm{LV}$ contractile reserve in univariable analysis: Age, sex, presence of ischemic heart disease (IHD), estimated glomerular filtration rate (eGFR), atrial fibrillation (AF) and DM status [3]. Further, the relationship between Gal-3, fibulin-1 and parameters of glucose metabolism as HbA1c and DM were examined in 
multivariable models including confounders associated with Gal-3 and fibulin- 1 at the $P<0.1$ level in univariable analysis. Multivariable linear regression analysis was performed as stepwise analyses with backward elimination. Logarithmic transformation of Gal-3 and fibulin-1 were used in the linear regression analyses to meet the assumptions of linearity. Statistical analyses were performed using the SPSS 22 package.

\section{Results}

According to the OGTT and history of DM, 70 (45\%) of the HF patients had NGT, 25 (16\%) had IGT, and 60 (39\%) DM, of these 28 (18\%) were newly diagnosed. Baseline characteristics according to DM status are presented in Table 1. There were no significant differences with regard to age, gender distribution, eGFR, and IHD between HF patients with and without DM. LVEF at rest $(P=0.003)$, LV contractile reserve $(P=0.01)$, and systolic longitudinal tissue velocity $\left(S^{\prime}\right)$ during $\operatorname{LDDE}(P=0.03)$ were reduced among patients with DM compared to the non-diabetic patients. Distributions of Gal-3 and of fibulin-1 are displayed in Fig. 1. Median (IQR) Gal-3 was $16.0(12.8-21.0) \mathrm{ng} / \mathrm{mL}$, and mean fibulin-1 was $57 \pm$ $16 \mu \mathrm{g} / \mathrm{mL}$. The univariable associations between the biomarkers and clinical parameters are shown in Table 2. Galectin-3 levels were strongly associated with impaired renal function (eGFR) $(\beta=-0.49, P<0.001)$ and $(\beta=-0.46, P<0.001)$ after full adjustment. Fibulin-1 levels were correlated to eGFR, NT-proBNP, presence of $\mathrm{AF}$, and age (Table 2) and remained correlated to eGFR $(\beta=-0.26, P<0.001)$ after full adjustment.

\section{Galectin-3, fibulin-1 and relationship with glucose metabolism}

Plasma Gal-3 levels were elevated among HF patients with DM (17.9 (12.9 26.1) ng/mL) and IGT (16.8 (14.122.1) $\mathrm{ng} / \mathrm{mL}$ ) as compared with NGT patients 15.2 $(11.6-18.3) \mathrm{ng} / \mathrm{mL}(P=0.02)$ (Table 1). No difference was found in patients classified as IGT compared to NGT $(P=0.38)$. The relationship between the presence of DM and Gal-3 levels was further investigated in a multivariable model adjusting for age, eGFR, AF, and NYHA functional class, being confounders associated with Gal-3 at the $P<0.1$ level. In this model DM was independently associated with elevated Gal-3 $(\beta=0.17$, $P=0.02$ ). An impact of glucose metabolism on Gal-3 levels was further observed by a positive association with HbA1c $(\beta=0.25, P=0.02)$ (Fig. 2$)$, and FPG $(P=0.02)$, but only borderline significantly associated with $2 \mathrm{hPG}$ and HOMA (Table 2). HbA1c was entered in a multivariable model, and demonstrated to have an independent relation with increasing Gal-3 levels $(\beta=0.16, P=0.02)$. After full adjustment, no association between Gal-3 and FPG $(P=0.56$, data not shown) was observed.
Table 1 Baseline characteristics according to diabetes status

\begin{tabular}{|c|c|c|c|}
\hline Demographics & $\mathrm{DM}(n=60)$ & Non-DM $(n=95)$ & $P$ value \\
\hline Age, years & $70.8(9.6)$ & $69.7(10.8)$ & 0.54 \\
\hline Sex (female/male), \% & $32 / 68$ & $33 / 67$ & 0.90 \\
\hline $\mathrm{HD}, \mathrm{n}(\%)$ & $40(67)$ & $52(55)$ & 0.20 \\
\hline NYHA I + II vs. III + IV, \% & $77 / 23$ & $85 / 15$ & 0.21 \\
\hline$A F, n(\%)$ & $18(30)$ & $21(22)$ & 0.30 \\
\hline Systolic BP, mmHg & $134(110-151)$ & $135(120-146)$ & 0.58 \\
\hline Heart rate, beats/min & $73(66-81)$ & $69(60-81)$ & 0.03 \\
\hline \multicolumn{4}{|l|}{ Metabolism } \\
\hline $\mathrm{BMI}, \mathrm{kg} / \mathrm{m}^{2}$ & $28.1(5.4)$ & $26.3(5.0)$ & 0.05 \\
\hline $\mathrm{FPG}, \mathrm{mmol} / \mathrm{L}$ & $6.9(1.4)$ & $5.7(0.5)$ & $<0.001$ \\
\hline $2 \mathrm{hPG}(\mathrm{mmol} / \mathrm{l})$ & $11.7(2.9)$ & $6.6(1.9)$ & $<0.001$ \\
\hline $\mathrm{HbA} 1 \mathrm{C}, \mathrm{mmol} / \mathrm{mol}$ & $49(12)$ & $40(4)$ & $<0.001$ \\
\hline HOMA-IR & $2.8(1.5-5.8)$ & $1.7(1.0-2,8)$ & $<0.001$ \\
\hline Total cholesterol, mmol/L & $4.0(1.0)$ & $4.4(1.1)$ & 0.02 \\
\hline Estimated GFR, ml/min/1.73 $\mathrm{m}^{2}$ & $68(26)$ & $74(19)$ & 0.10 \\
\hline \multicolumn{4}{|l|}{ Biomarkers } \\
\hline NT-proBNP, pg/mL & $1111(418-2227)$ & $771(310-1854)$ & 0.08 \\
\hline Gal-3, ng/ml & $17.9(12.9-26.1)$ & $15.6(12.6-19.3)$ & 0.02 \\
\hline Fibulin-1, $\mu \mathrm{g} / \mathrm{ml}$ & $60(19)$ & $55(13)$ & 0.07 \\
\hline \multicolumn{4}{|l|}{ Echocardiographic variables } \\
\hline LVEF rest, \% & $34(29-41)$ & $39(33-45)$ & 0.003 \\
\hline$S^{\prime}$ (rest), $\mathrm{cm} / \mathrm{s}$ & $5.2(4.5-6.5)$ & $5.7(4.8-6.4)$ & 0.10 \\
\hline $\mathrm{E}, \mathrm{cm} / \mathrm{s}$ & $81.6(62.2-99.7)$ & $72.9(60.0-92.4)$ & 0.31 \\
\hline$e^{\prime}, \mathrm{cm} / \mathrm{s}$ & $5.9(4.6-7.5)$ & $6.6(5.5-8.7)$ & 0.03 \\
\hline E/e' (rest) per unit & $12.2(9.6-16.5)$ & $10.2(8.1-15.4)$ & 0.007 \\
\hline LVEF stress, \% & $40(34-47)$ & $49(40-57)$ & $<0.001$ \\
\hline LV contractile reserve, $\%$ & $7(2-10)$ & $10(4-15)$ & 0.01 \\
\hline $\mathrm{S}^{\prime}$ (stress), $\mathrm{cm} / \mathrm{s}$ & $8.3(6.3-9.6)$ & $7.3(5.7-8.5)$ & 0.03 \\
\hline \multicolumn{4}{|l|}{ Medical treatment } \\
\hline ACE/Angll-inhibitors, n, (\%) & $85(89)$ & $48(80)$ & 0.10 \\
\hline$\beta$-blocker, n (\%) & $53(88)$ & $72(76)$ & 0.05 \\
\hline Loop diuretic dose, mg/24 h & $60(0-80)$ & $0(0-60)$ & 0.001 \\
\hline
\end{tabular}

Oral glucose tolerance test was only performed in the non-diabetic $\mathrm{HF}$ patient. 2hPG: $2 \mathrm{~h}$ plasma glucose. AF Atrial fibrillation; BMI Body mass index; BSA Body surface area $E$ Peak early diastolic transmitral flow velocity; $e^{\prime}$; early diastolic longitudinal tissue velocities; FPG Fasting plasma glucose; Gal-3 Galectin-3; GFR Glomerular filtration rate; HOMA-IR Homeostatic model assessment of Insulin Resistance. IHD Ischemic heart disease; $L V$ left ventricular; $L V E F$ Left ventricular ejection fraction; NT-proBNP N-terminal pro B-natriuretic peptide; NYHA NYHA functional class; $S^{\prime}$ Systolic longitudinal tissue velocity

There was only a trend towards increased plasma fibulin-1 levels when comparing in HF patients with DM vs non DM $(P=0.07)$, although impaired glucose metabolism assessed by presence of IGT and DM were associated with equivalent elevated mean fibulin-1 levels $61 \pm 16 \mu \mathrm{g} / \mathrm{mL}$ vs. $60 \pm 19 \mu \mathrm{g} / \mathrm{mL}$, respectively compared to HF patients with NGT $52 \pm 13 \mu \mathrm{g} / \mathrm{mL}(P=0.01)$. Further, $\mathrm{HbA1c}$ was associated with increasing fibulin-1 


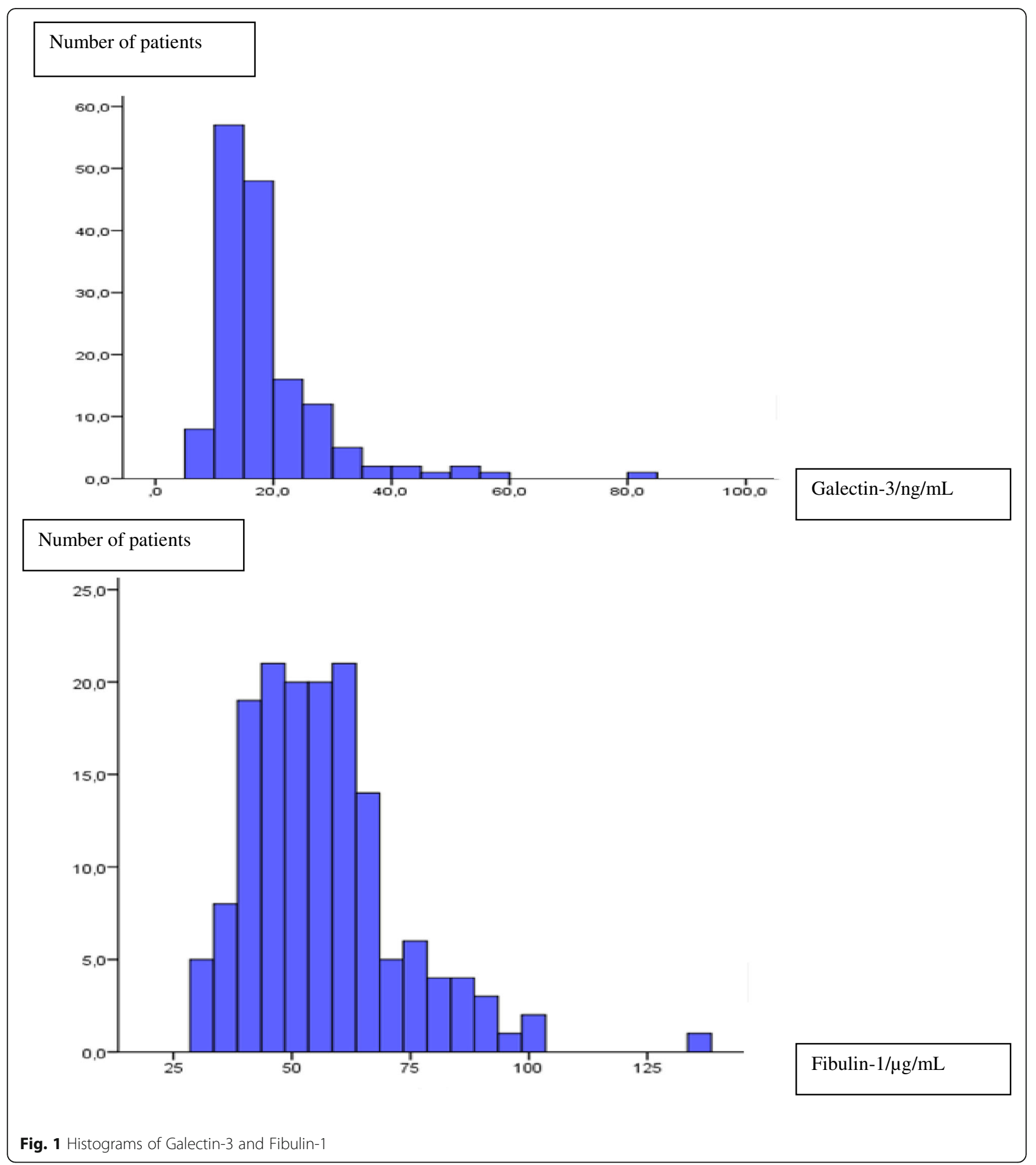

concentrations ( $\beta=0.21, P=0.009$ ) (Fig. 2), and this relation was not markedly attenuated after adjustment for the confounders age, AF, and eGFR $(\beta=0.15, P=0.05)$. Fibulin-1 levels which were more than 2SD from mean Finulin-1 levels were considered outliers. Hence, we excluded 3 measurements of Fibulin-1 levels which were considered outliers. The exclusion of the outliers did not change the results (data not shown).

\section{Galectin-3, fibulin-1 and relationship with LV function}

The univariable associations between plasma Gal-3 and fibulin-1 levels with echocardiographic parameters 
Table 2 Associations of galectin-3 and fibulin-1 levels with clinical and glucometabolic parameters

\begin{tabular}{|c|c|c|c|c|}
\hline \multirow[b]{2}{*}{ Parameter } & \multicolumn{2}{|l|}{ Galectin-3 } & \multicolumn{2}{|l|}{ Fibulin-1 } \\
\hline & $\beta(\mathrm{SE})$ & $P$-Value & $\beta$ (SE) & $P$-Value \\
\hline Age, per year & $0.16(0.23)$ & 0.05 & $0.36(0.12)$ & $<0.001$ \\
\hline Sex & $0.04(0.07)$ & 0.59 & $-0.09(2.75)$ & 0.29 \\
\hline Ischemic heart disease & $-0.01(0.07)$ & 0.90 & $-0.009(2.71)$ & 0.92 \\
\hline NYHA I + II vs. III + IV & $0.16(0.09)$ & 0.06 & $0.08(3.37)$ & 0.34 \\
\hline Atrial fibrillation & $0.17(0.07)$ & 0.03 & $0.17(2.83)$ & 0.03 \\
\hline Diabetes mellitus & $0.27(0.07)$ & 0.001 & $0.16(2.62)$ & 0.05 \\
\hline eGFR, per $\mathrm{ml} / \mathrm{min} / 1.73 \mathrm{~m}^{2}$ & $-0.49(0.10)$ & $<0.001$ & $-0.33(0.06)$ & $<0.001$ \\
\hline \multicolumn{5}{|l|}{ Metabolism } \\
\hline BMl, per kg/m² & $-0.04(0.006)$ & 0.63 & $-0.12(0.25)$ & 0.14 \\
\hline FPG, per $\mathrm{mmol} / \mathrm{L}$ & $0.19(0.02)$ & 0.02 & $-0.07(1.37)$ & 0.44 \\
\hline 2hPG, per mmol/L & $0.15(0.01)$ & 0.09 & $0.26(0.42)$ & 0.004 \\
\hline $\mathrm{HbA} 1 \mathrm{C}$, per $\mathrm{mmol} / \mathrm{mol}$ & $0.25(0.16)$ & 0.02 & $0.21(0.14)$ & 0.009 \\
\hline HOMA-IR, per unit & $0.17(0.01)$ & 0.06 & $-0.08(0.52)$ & 0.36 \\
\hline \multicolumn{5}{|l|}{ Biomarkers } \\
\hline NT-proBNP per pg/mL & $0.30(0.12)$ & $<0.001$ & $0.38(2.24)$ & $<0.001$ \\
\hline
\end{tabular}

obtained at rest and during LDDE are displayed in Table 3. There was an association between Gal-3 and LV contractile reserve $(\beta=-0.19, P=0.03)$ and $G a l-3$ and LVEF during stress $(\beta=-0.25, P=0.003)$. In a multivariable regression model the association with LV contractile reserve was no longer significant $(P=0.66)$, as only DM $(\beta=-0.20, P=0.03)$ and eGFR $(\beta=-0.18, P=0.04)$ remained predictive of $\mathrm{LV}$ contractile reserve after complete adjustment (data not shown).

Finally, the patients were divided into two groups; normal and low eGFR $\left(<60 \mathrm{~mL} / \mathrm{min} / 1.73 \mathrm{~m}^{2}\right)$. In both groups, no association between Gal-3 and LV contractile reserve $(\beta=-0.06, P=0.57$ in patients with normal eGFR vs. $\beta=-0.02, P=0.95$ in patients with low eGFR) was demonstrated. With regard to fibulin-1, resting LVEF, LVEF and S' during stress were all associated with increasing levels of this biomarker in a univariable analysis, as presented in Table 3. Yet, after adjustment for relevant co-variables including eGFR no independent associations between fibulin-1 and these LV systolic measures were found. An independent relation of fibulin-1 with decreasing eGFR $(\beta=-0.24, P=0.004)$ and with age $(\beta=0.22, P=0.008)$ was observed. Again, the patients were divided into two groups; normal and low eGFR $\left(<60 \mathrm{~mL} / \mathrm{min} / 1.73 \mathrm{~m}^{2}\right)$. In both groups, no association between fibulin-1 and LV contractile reserve ( $\beta=0.06, P=0.55$ in patients with normal eGFR vs. $\beta=$ $0.02, P=0.89$ in patients with low eGFR) was presented. We have performed analyses where the patients with known diabetes were separated from the patients with newly diagnosed diabetes. This, however, did not influence the results.

\section{Discussion}

The primary findings of the present study were that plasma Gal-3 and fibulin-1 levels were elevated in HFrEF patients with abnormal glucose metabolism compared to HFrEF patients with NGT. The impact of impaired glucose metabolism on both Gal-3 and fibulin-1 levels was underlined by an independent association with increasing HbA1c. However, increased Gal-3 and fibulin-1 levels were not associated with the reduced contractile reserve observed among diabetic HFrEF patients.

Median Gal-3 level was $16.0 \mathrm{ng} / \mathrm{mL}$, which is similar to findings in comparable HFrEF populations and clearly increased compared to healthy subjects $[17,18]$. The mean fibulin-1 level in our HF population was $57 \pm 16 \mu \mathrm{g} / \mathrm{mL}$, which is slightly higher than the mean level observed in a DM population (46 $\pm 1.3 \mu \mathrm{g} / \mathrm{mL}$ [19]). Fibulin-1 is a novel biomarker and high concentrations have primarily been reported in patients with aortic stenosis and DM compared to a level of $5.6(4.1-8.4) \mu \mathrm{g} / \mathrm{mL}$ reported in healthy men $[11,20,21]$.

The current results suggest that glucose metabolism is associated with circulating Gal-3 concentrations in HF, as elevated levels were found in patients with DM and an association with increasing $\mathrm{HbA} 1 \mathrm{c}$ was demonstrated. The observations were supported by two previous 
Log Fibulin-1

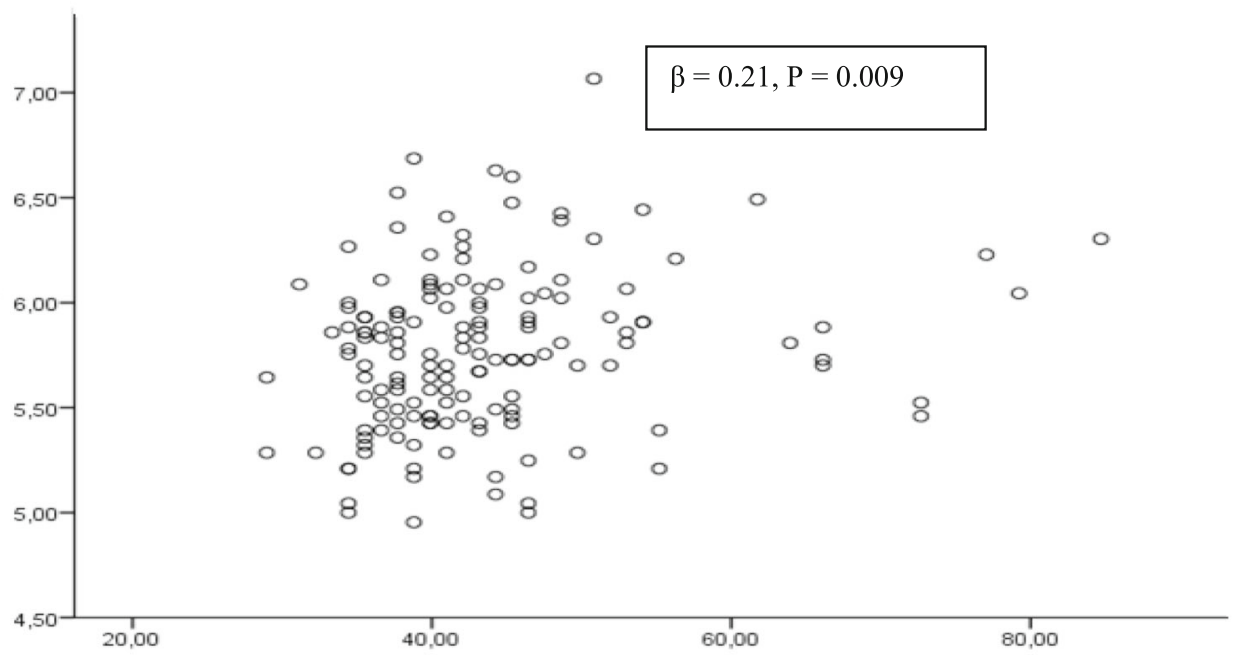

$\mathrm{HbAlc}, \mathrm{mmol} / \mathrm{mol}$

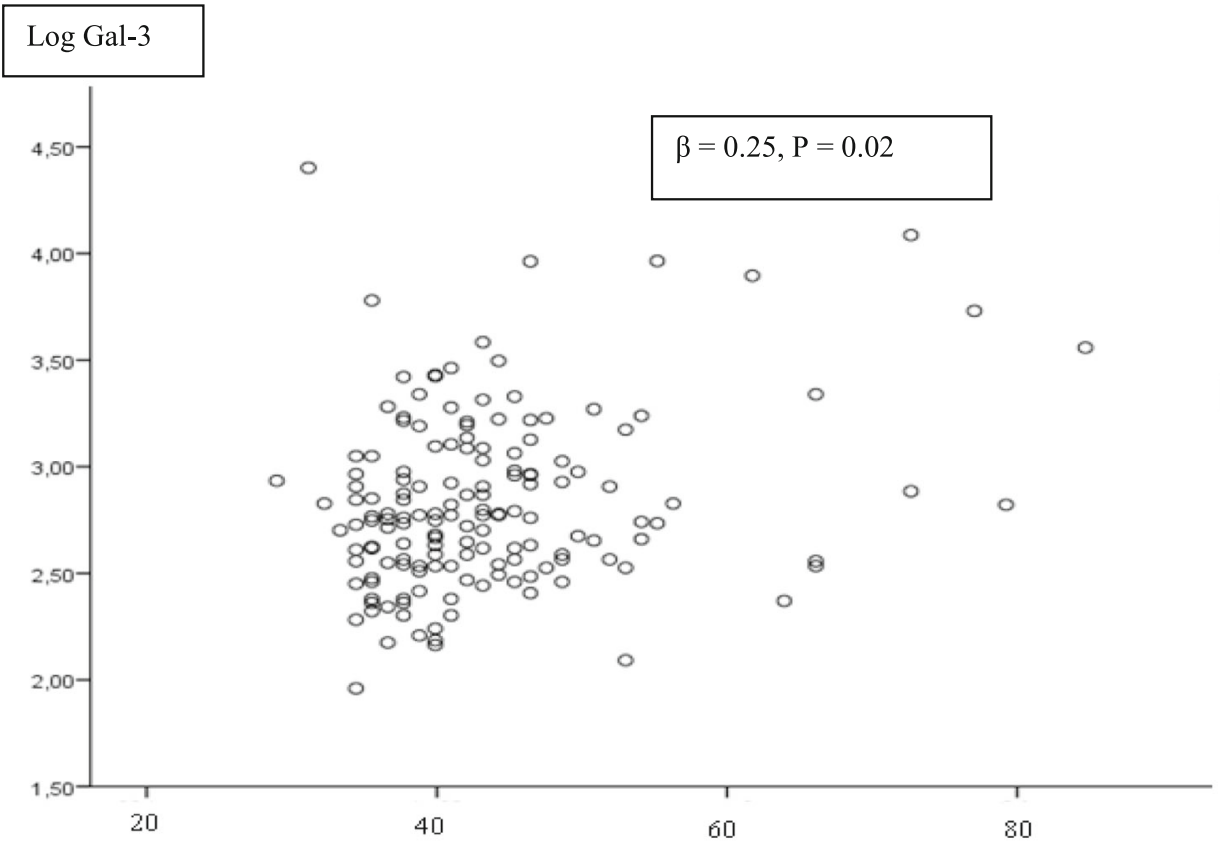

$\mathrm{HbAlc}, \mathrm{mmol} / \mathrm{mol}$

Fig. 2 Scatter plot of HbA1c, Galectin-3 and fibulin-1 levels

studies reporting increased concentrations in patients with type 2 DM $[18,21]$. However, we found a significant impact of renal function on plasma Gal-3 levels suggesting that other parameters, such as DM complications like diabetic nephropathy were more predictive of Gal-3 levels in HFrEF patients.

With regard to fibulin-1 we found elevated concentrations among patients with IGT and DM compared to NGT and a positive association with HbA1c, which corresponds with previous findings among type 2 DM reporting elevated circulating levels and of an upregulation of fibulin-1 in the arterial walls [19, 22].

To our knowledge, the present study is the first to evaluate the relation between circulating Gal-3 and fibulin-1 levels and cardiac function evaluated by LDDE. Galectin-3 was not associated with LV contractile reserve and LVEF during low dose dobutamine infusion after adjusting for relevant confounders, especially eGFR. The present study demonstrated that impaired renal function was a strong and independent predictor of 
Table 3 Associations of galectin-3 and fibulin-1 levels with echocardiographic parameters

\begin{tabular}{|c|c|c|c|c|}
\hline & \multicolumn{2}{|l|}{ Galectin-3 } & \multicolumn{2}{|l|}{ Fibulin-1 } \\
\hline & $\beta(\mathrm{SE})$ & $P$-Value & $\beta(S E)$ & $P$-Value \\
\hline \multicolumn{5}{|l|}{ Resting echocardiography } \\
\hline LVEF, per \% & $-0.15(0.14)$ & 0.08 & $-0.24(0.14)$ & 0.004 \\
\hline $\mathrm{S}^{\prime}$, per $\mathrm{cm} / \mathrm{s}$ & $-0.15(0.14)$ & 0.07 & $-0.25(0.02)$ & 0.002 \\
\hline \multicolumn{5}{|l|}{ Stress echocardiography } \\
\hline LVEF stress, per \% & $-0.25(0.14)$ & 0.003 & $-0.20(0.003)$ & 0.02 \\
\hline LV contractile reserve, per \% & $-0.19(0.06)$ & 0.03 & $-0.03(0.005)$ & 0.71 \\
\hline $\mathrm{S}^{\prime}$ (stress), per $\mathrm{cm} / \mathrm{s}$ & $-0.15(0.14)$ & 0.07 & $-0.24(0.01)$ & 0.004 \\
\hline
\end{tabular}

Univariable regression analyses

$L V$ left ventricular/left ventricle, $L V E F$ Left ventricular ejection fraction, $S^{\prime}$ Systolic longitudinal tissue velocity

elevated Gal-3 levels, which is in line with previous studies in patients with DM and in a community cohort $[8,23]$. The elimination of Gal-3 has to our best knowledge not been described, but the present findings indicate a renal component. The gold standard for evaluation of myocardial fibrosis requires an endomyocardial biopsy, which is a high-risk procedure. Cardiac magnetic resonance imaging (CMR) is another method that seems to provide a good estimate of myocardial fibrosis [24, 25]. However, it is technically difficult to perform CMR in patients with $\mathrm{AF}$ and devices; which are highly prevalent in HFrEF patients. Furthermore, the association between cardiac fibrosis estimated by CMR and Gal-3 levels is not well described. In patients with previous myocardial infarction Gal-3 was moderately associated with LVEF and infarct size [26]. We report no association of the biomarkers Gal-3 with IHD. A proposed mechanism could be that former MI in our HFrEF patients are not associated with persisting increased activity of the fibrotic tissue and thereby secretion to the circulation of these biomarkers.

Myocardial fibrosis evaluated by histomorphometry and LV contractile reserve seems associated in patients with dilated cardiomyopathy [5]. In patients with acute decompensated HF, the PRIDE study reported a relationship between Gal-3 and resting echocardiographic parameters related to diastolic function [27]. In the current cohort of patients with chronic HFrEF we found no independent association with parameters related to diastolic function (data not shown).

We found no association between fibulin-1 levels and LV contractile reserve, while univariable associations were demonstrated between fibulin-1 and resting parameters LVEF and S'. However, no association was shown in the multivariable analyses. This is partly in accordance with data from patients with aorta stenosis, where fibulin-1 levels were related with S', but not with LVEF [10]. Furthermore, we demonstrated a moderate, inverse association with eGFR which could blunt a potential weaker association with $\mathrm{LV}$ contractile reserve.

\section{Limitations}

This is a cross-sectional study. Hence, conclusion regarding causality is not possible. A larger sample size would have allowed us to investigate the IGT group in more detail.

\section{Conclusion}

Galectin-3, but not fibulin-1 levels are elevated in diabetic compared to non-diabetic HFrEF patients. Both Gal-3 and fibulin-1 levels are independently and positively associated with $\mathrm{HbA1c}$, but not with LV contractile reserve in HF patients.

\section{Abbreviations}

2hPG: 2 h plasma glucose; AF: Atrial fibrillation; BMl: Body mass index; BSA: Body surface area; CMR: Cardiac magnetic resonance imaging; DM: Diabetes mellitus; E: Peak early diastolic transmitral flow velocity; e': Early diastolic longitudinal tissue velocities; eGFR: Estimated glomerular filtration rate; FPG: Fasting plasma glucose; Gal-3: Galectin 3; GFR: Glomerular filtration rate; HF: Heart failure; HFrEF: Heart failure with reduced ejection fraction; HOMA-IR: Homeostasis Model of Assessment-Insulin Resistance; IGT: Impaired glucose tolerance; IHD: Ischemic heart disease; LDDE: Low dose dobutamine echocardiography; LV: Left ventricular; LVEF: Left ventricular ejection fraction; MI: Myocardial infarction; NGT: Normal Glucose tolerance; NT-proBNP: Nterminal pro B-natriuretic peptide; NYHA: NYHA functional class; OGTT: Oral Glucose Tolerance Test; S': Systolic longitudinal tissue velocity; SD: Standard deviation

\section{Acknowledgements}

Ulla Kjaerulff-Hansen, Hanne Dorthe Mogensen, Susanne Månsson, Marianne Sørensen and Anne-Marie Jakobsen are thanked for assistance with biomarker analyses. Department of Clinical Biochemistry at Frederiksberg Hospital is thanked for conducting the OGTTs.

\section{Funding}

The study was financed from a research grant from Herlev University Hospital.

\section{Availability of data and materials}

The dataset supporting this study is part of a larger dataset of the cohort from the heart failure outpatient clinic, Frederiksberg Hospital. Questions concerning the dataset supporting the conclusions of this article can be addressed to the main author.

\section{Authors' contributions}

PH: Acquisition of data, interpretation of data, drafting the manuscript, final approval of the version. ME: acquisition of data, analysis of data, final approval of the version. IG: hypothesis generating, revising manuscript critically for important intellectual content, final approval of the version. MS: Interpretation of data, final approval of the version. JD and LMR: interpretation of data 
(especially regarding fibulin-1), final approval of the version. JME and CDT: interpretation of data, revising manuscript critically, final approval of the version. JF and CK: Hypothesis generating, revising manuscript critically for important intellectual content, final approval of the version. All authors read and approved the final manuscript.

\section{Competing interests}

None of the authors have reported any conflicts of interest with regard to the present study.

\section{Consent for publication}

Not applicable.

\section{Ethics approval and consent to participate}

The study was approved by the local ethics committee in The Capital Region, Denmark, and The National Board of Registration and was conducted according to the Declaration of Helsinki. All patients provided written informed consent.

\section{Author details}

${ }^{1}$ Department of Medicine, Endocrine Unit, Herlev University Hospital, Herlev Denmark. 'Faculty of Health and Medical Sciences, Copenhagen University, Copenhagen, Denmark. ${ }^{3}$ Department of Cardiology, Rigshospitalet, Copenhagen, Denmark. ${ }^{4}$ Department of Cardiology, Hvidovre University Hospital, Hvidovre, Denmark. ${ }^{5}$ Department of Cardiology, Herlev University Hospital, Herlev, Denmark. ${ }^{6}$ Department of Cardiology and Cardiothoracic Surgery, Odense University Hospital, Odense, Denmark. ${ }^{7}$ Department of Clinical Biochemistry and Pharmacology, Odense University Hospital, Odense, Denmark. ${ }^{8}$ Centre of Individualized Medicine in Arterial Diseases, Odense University Hospital, Odense, Denmark. ${ }^{9}$ Department of Cardiology, Odense University Hospital, Odense, Denmark. ${ }^{10}$ Department of Cardiology, Bispebjerg University Hospital, Copenhagen, Denmark. ${ }^{11}$ Department of Endocrinology, Herlev Hospital, Herlev, Denmark.

Received: 9 September 2016 Accepted: 7 December 2016 Published online: 10 January 2017

\section{References}

1. Gustafsson I, Brendorp B, Seibaek M, et al. Influence of diabetes and diabetes-gender interaction on the risk of death in patients hospitalized with congestive heart failure. J Am Coll Cardiol. 2004;43:771-7.

2. Egstrup M, Schou M, Gustafsson I, et al. Oral glucose tolerance testing in an outpatient heart failure clinic reveals a high proportion of undiagnosed diabetic patients with an adverse prognosis. Eur J Heart Fail. 2011;13(3):319-26.

3. Egstrup M, Kistorp CN, Schou M, et al. Abnormal glucose metabolism is associated with reduced left ventricular contractile reserve and exercise intolerance in patients with chronic heart failure. Eur Heart J Cardiovasc Imaging. 2013;14(4):349-57.

4. Williams MJ, Odabashian J, Lauer MS, et al. Prognostic value of dobutamine echocardiography in patients with left ventricular dysfunction. J Am Coll Cardiol. 1996:27(1):132-9.

5. Otasevic P, Popovic ZB, Vasiljevic JD, et al. Relation of myocardial histomorphometric features and left ventricular contractile reserve assessed by high-dose dobutamine stress echocardiography in patients with idiopathic dilated cardiomyopathy. Eur J Heart Fail. 2005;7(1):49-56.

6. Sharma UC, Pokharel S, van Brakel TJ, et al. Galectin-3 marks activated macrophages in failure-prone hypertrophied hearts and contributes to cardiac dysfunction. Circulation. 2004;110(19):3121-8.

7. Jin $\mathrm{QH}$, Lou YF, Li TL, et al. Serum galectin-3: a risk factor for vascular complications in type 2 diabetes mellitus. Chin Med J (Engl) 2013;126(11):2109-15.

8. Gopal DM, Kommineni M, Ayalon $\mathrm{N}$ et al. Relationship of plasma galectin-3 to renal fuinction in patients with heart failure: effects of clinical status, pathophysiology of heart failure, and presence or abscence of heart failure. J Am Heart Assoc. 2012;1(5):e000760. doi: 10.1161/JAHA.112.000760. Epub 2012 Oct 25

9. Beisvag V, Lehre PK, Midelfart H, et al. Aetiology-sepcific patterns in endstage heart failure patients identified by functional annotation and classification of microarray data. Eur J Heart Fail. 2006;8:381-9.
10. Hansen ML, Dahl JS, Argraves WS, et al. Aortic valve stenosis and atrial fibrillation influence plasma fibulin-1 levels in patients treated with coronary bypass surgery. Cardiology. 2013;126(3):202-6.

11. Schoze A, Bladbjerg EM, Sidelmann Jj et al. Plasma concentrations of extracellular matrix protein fibulin-1 are related to cardiovascular risk markers in chronic kidney disease and diabetes. Cardiovascl. Diabetol 2013 Jan 7:12:6. Doi: 10.1186/1475-2840-12-6

12. Dahl JS, Moller JE, Videbaek $L$, et al. Plasma fibulin-1 is linked to restrictive filling of the left ventricle and to mortality in patients with aortic valve stenosis. J Am Heart Assoc. 2012;1(6):e003889.

13. Lang RM, Bierig M, Devereux RB, et al. Recommendations for chamber quantification. Eur J Echocardiogr. 2006;7(2):79-108.

14. Christenson $\mathrm{RH}$, Duh $\mathrm{SH}, \mathrm{Wu} \mathrm{AH}$, et al. Multi-center determination of galectin-3 assay performance characteristics: Anatomy of a novel assay for use in heart failure. Clin Biochem. 2010;43(7-8):683-90.

15. Cangemi C, Skov V, Poulsen MK, et al. Fibulin-1 is a marker for arterial extracellular matrix alterations in type 2 diabetes. Clin Chem. 2011:57(11):1556-65.

16. Argraves WS, Tran $H$, Burgess WH, et al. Fibulin is an extracellular matrix and plasma glycoprotein with repeated domain structure. J Cell Biol. 1990;111(6 Pt 2):3155-64

17. Tang WH, Shrestha $K$, Shao Z, et al. Usefulness of plasma galectin-3 levels in systolic heart failure to predict renal insufficiency and survival. Am J Cardiol. 2011:108(3):385-90.

18. Weigert J, Neumeier M, Wanninger J, et al. Serum galectin-3 is elevated in obesity and negatively correlates with glycosylated hemoglobin in type 2 diabetes. J Clin Endocrinol Metab. 2010;95(3):1404-11.

19. Skov V, Cangemi C, Gram J, et al. Metformin, but not rosiglitazone, attenuates the increasing plasma levels of a new cardiovascular marker, fibulin-1, in patients with type 2 diabetes. Diabetes Care. 2014;37(3):760-6.

20. Hansen ML, Rasmussen LM. Associations between plasma fibulin-1, pulse wave velocity and diabetes in patients with coronary heart disease. J Diabetes Complications. 2015:29(3):362-6. doi:10.1016/j.jdiacomp.2015. 01.003. Epub 2015 Jan 13.

21. Paapstel K, Zilmer M, Eha J, et al. Association between fibulin-1 and aortic augmentation index in male patients with peripheral arterial disease. Eur Vasc Endovasc Surg. 2016:51(1):76-82. doi:10.1016/j.ejvs.2015.09.004. Epub 2015 Oct 21.

22. Skov V, Knudsen S, Olesen $\mathrm{M}$, et al. Global gene expression profiling displays a network of dysregulated genes in non-atherosclerotic arterial tissue from patients with type 2 diabetes. Cardiovasc Diabetol. 2012;11:15.

23. Ho JE, Liu C, Lyass A, et al. Galectin-3, a marker of cardiac fibrosis, predicts incident heart failure in the community. J Am Coll Cardiol. 2012:60(14):1249-56.

24. Mewton N, Liu CY, Croisille P, et al. Assessment of myocardial fibrosis with cardiovascular magnetic resonance. J Am Coll Cardiol. 2011:57(8):891-903.

25. Karamitsos TD, Francis JM, Myerson S, et al. The role of cardiovascular magnetic resonance imaging in heart failure. J Am Coll Cardiol. 2009:54(15):1407-24.

26. Singsaas EG, Manhenke CA, Dickstein $K$, et al. Circulation Galectin-3 levels are increased in patients with ischemic heart disease, but are not influenced by acute myocardial infarction. Cardiology. 2016;134:398-405. doi:10.1159/ 000445103.

27. Shah RV, Chen-Tournoux AA, Picard MH, et al. Galectin-3, cardiac structure and function, and long-term mortality in patients with acutely decompensated heart failure. Eur J Heart Fail. 2010;12(8):826-32.

\section{Submit your next manuscript to BioMed Central and we will help you at every step:}

- We accept pre-submission inquiries

- Our selector tool helps you to find the most relevant journal

- We provide round the clock customer support

- Convenient online submission

- Thorough peer review

- Inclusion in PubMed and all major indexing services

- Maximum visibility for your research

Submit your manuscript at www.biomedcentral.com/submit
Biomed Central 